\title{
Mudanças na prevalência de excesso de peso em adolescentes residentes em área de alta vulnerabilidade a insegurança alimentar
}

\author{
Changes in prevalence of overweight in adolescents \\ living in areas highly vulnerable to food insecurity
}

Danilo Dias Santana (https://orcid.org/0000-0002-9769-7435) ${ }^{1}$

Erica Guimarães Barros (https://orcid.org/0000-0001-5083-2399) ${ }^{1}$

Rosana Salles-Costa (https://orcid.org/0000-0002-2307-4083) ${ }^{1}$

Gloria Valeria da Veiga (https://orcid.org/0000-0002-7985-0213) ${ }^{1}$

${ }^{1}$ Instituto de Nutrição Josué de Castro, Universidade Federal do Rio de Janeiro. Av. Carlos Chagas Filho 373, Bloco J, $2^{\circ}$ andar, Cidade Universitária. 21941-902 Rio de Janeiro RJ Brasil. dias.danilo@hotmail.com

\begin{abstract}
This study ascertained changes, over 5 years, in the prevalence of overweight in adolescents and associations with socio-demographic variables and food insecurity. Two cross-sectional population-based studies were conducted with 511 (2005) and 314 (2010) adolescents resident in Campos Elíseos (Duque de Caxias-RJ). Overweight was evaluated by sex and age specific cut-off points of BMI (weight/height ${ }^{2}$ ). The prevalence of food insecurity was investigated using the Brazilian Food Insecurity Scale. Logistic regression was used to determine the association between changes in overweight over time and sex, age, skin colour, and food insecurity. Overweight was found to increase significantly, between 2005 and 2010, in boys who were younger (from 20.1\% to $49.5 \%)$, black or brown (22.2\% to $37.3 \%$ ), those with per capita income of up to half a minimum wage (13.6\% to $32.5 \%$ ) and those experiencing moderate or severe food insecurity $(9.2 \%$ to $36.3 \%)$. It was concluded that overweight increased significantly in adolescents living in an area of food insecurity, and that younger, black or brown, lower-income adolescents, and those living with moderate and severe food insecurity, were more exposed to that increase.
\end{abstract}

Key words Overweight, Obesity, Food and nutrition security, Adolescent
Resumo $O$ objetivo deste estudo foi verificar as mudanças na prevalência do excesso de peso em adolescentes e sua associação com variáveis sociodemográficas e insegurança alimentar em período de 5 anos. Foram realizados dois estudos transversais com 511 (2005) e 314 (2010) adolescentes de 12 a 18,9 anos residentes de Campos Elíseos (Duque de Caxias-RJ). Excesso de peso foi avaliado por meio do IMC (peso/estatura ${ }^{2}$ ). A insegurança alimentar foi investigada por meio da Escala Brasileira de Insegurança Alimentar (EBIA). Utilizou-se regressão logística para verificar a associação entre as mudanças temporais de excesso de peso segundo sexo, idade, cor da pele, e insegurança alimentar. Quanto à evolução do excesso de peso pode se notar que nos meninos houve aumento significativo para os mais novos $(20,1 \%$ para $49,5 \%)$, os de cor preta ou parda (22,2\% para $37,3 \%)$, os que tinham renda per capita de até $1 / 2$ salário minimo (13,6\% para $32,5 \%)$ e os que apresentavam insegurança alimentar moderada e grave $(9,2 \%$ para 36,3\%) entre 2005 e 2010. Conclui-se que o aumento do excesso de peso foi expressivo nos adolescentes residentes em área de insegurança alimentar, e os meninos mais novos, pretos ou pardos, de menor renda e residentes em lares com insegurança alimentar moderada e grave estão mais expostos a este aumento.

Palavras-chave Sobrepeso, Obesidade, Segurança alimentar e nutricional, Adolescente 


\section{Introdução}

O aumento significativo na prevalência de excesso de peso no Brasil tem sido evidente ${ }^{1,2}$, seguindo uma tendência mundial ${ }^{3}$. Entre os adolescentes o excesso de peso aumentou, aproximadamente, seis vezes no sexo masculino (de 3,7\% para $21,7 \%$ ) e quase três vezes no sexo feminino (de $7,6 \%$ para $19,4 \%$ ) no período de 1974 a 2009 , e esta tendência ascendente também se observou para obesidade ${ }^{1}$, caracterizando-a como um grave problema de saúde pública. Esta situação quando presente em um contexto de evidente insegurança alimentar e nutricional é intrigante e gera questionamento sobre as possíveis associações entre os dois problemas.

Segurança Alimentar e Nutricional (SAN) é definida como a realização do direito de todos ao acesso, regular e permanente, a alimentos de qualidade, em quantidade suficiente, sem comprometer $o$ acesso a outras necessidades essenciais, tendo como base práticas alimentares promotoras de saúde, que respeitem a diversidade cultural e que sejam socioeconômica e ambientalmente sustentáveis. Situações de insegurança alimentar podem ser detectadas por diferentes tipos de problemas tais como fome, consumo de alimentos de qualidade duvidosa ou prejudicial à saúde, com consequente obesidade e outras doenças associadas à má alimentação, entre outras ${ }^{5}$.

Estudos internacionais com crianças e adolescentes, mostraram que a insegurança alimentar esteve diretamente associada ao excesso de peso ${ }^{6-9}$, como também a uma dieta de baixa qualidade, caracterizada pela presença de alimentos menos saudáveis com maior probabilidade de levar a deficiência de nutrientes ${ }^{10,11}$. No Brasil, esta associação já foi observada em adultos ${ }^{12}$, entretanto ainda não está bem esclarecida em adolescentes, sendo encontrada associação inversa ${ }^{13}$ ou nula ${ }^{14,15}$.

Salles-Costa et al. ${ }^{16}$, em estudo realizado em 2005, no distrito de Campos Elíseos, Duque de Caxias-RJ, notaram que a insegurança alimentar estava presente em 53,8\% dos domićlios em diferentes graus $(31,4 \%$ com insegurança alimentar leve, $16,1 \%$ insegurança alimentar moderada e $6,3 \%$ insegurança alimentar grave). Por outro lado, Ferreira et al. ${ }^{17}$ divulgaram que $16,8 \%$ dos adolescentes que residiam em domicílios com insegurança alimentar apresentavam sobrepeso e $7,2 \%$ estavam obesos. Com a realização de nova pesquisa em Campos Elíseos, em 2010, no qual se reavaliou os aspectos abordados na pesquisa de $2005^{16}$, questionou-se como teria evoluído a prevalência de excesso de peso em adolescentes no período entre os dois estudos.

Sendo assim, objetivo deste artigo foi verificar as mudanças na prevalência do excesso de peso em adolescentes residentes em Campos Elíseos, Duque de Caxias, e sua associação com fatores sociodemográficos e insegurança alimentar, em período de 5 anos.

\section{Métodos}

O presente trabalho utiliza dados de dois estudos transversais, de base populacional, realizados em 2005 e 2010, por meio de visitas domiciliares, nas quais se investigaram variáveis referentes aos residentes em domicílios particulares permanentes (DPPs) do segundo distrito (Campos Elíseos) do município de Duque de Caxias, no estado do Rio de Janeiro. Na época do segundo estudo (2010), Duque de Caxias possuía população, predominantemente, urbana, estimada em 855.048 habitantes, com Índice de Desenvolvimento Humano Municipal (IDHM) de 0,711. Em 2005, Campos Elíseos destacava-se por ser um dos distritos de menor renda per capita de Duque de Caxias, com apenas $26,8 \%$ das famílias com renda acima de um salário mínimo, cerca de $83 \%$ das famílias classificadas nas classes sociais $\mathrm{C}$ e $\mathrm{D}$, segundo a classificação da ABIPEME, e 52,3\% dos chefes de família não concluintes do ensino fundamental. Mais informações sobre o município podem ser consultadas em Salles-Costa et al. ${ }^{16}$.

Os estudos foram realizados com amostras probabilísticas de 1.125 DPPs selecionados em três estágios (setor censitário, domicílio e o indivíduo). O tamanho da amostra foi fixado, tanto em 2005 como em 2010, com base em uma estimativa de $14,5 \%$ para a prevalência de pobreza extrema, fixando-se um erro relativo máximo de $5 \%$. Em 2005, no primeiro estágio, foram selecionados, de forma sistemática, 75 setores censitários com probabilidades proporcionais ao número de DPPs observados pelo Censo Demográfico 2000, dentre os 322 setores constantes da Base Operacional Geográfica (BOG) de 2000 do IBGE. Informações detalhadas quanto aos critérios de amostragem podem ser encontradas em Salles-Costa et al. ${ }^{16}$.

No segundo inquérito, realizado no ano de 2010, optou-se pelo desenho de amostragem invers ${ }^{18}$, por conglomerados (setor censitário, domicílios e indivíduos). No primeiro estágio de seleção, os mesmos 75 setores censitários foram amostrados, sendo realizado novo screening para 
atualização dos DPPs por estratos etários. Com esta informação, sorteou-se, de modo aleatório, os DPPs do estrato de crianças (máximo de oito domicílios por setor) seguidos pelo sorteio de DPPs com adultos e/ou adolescentes até completar o total de 15 DPPs por setor (segundo estágio de seleção).

O terceiro estágio contemplou a seleção dos indivíduos de cada DPP a serem avaliados, sendo selecionado um indivíduo de cada faixa etária (criança, adolescente ou adulto) e no estrato de DPPs só com adultos foram selecionados dois adultos. Os adultos entrevistados sobre as condições socioeconômicas das famílias foram a mulher de referência, ou seja, aquela responsável pelo gerenciamento das compras e preparo das refeições e o seu cônjuge, exceto quando a mulher não morava com seu companheiro ou quando este não estava presente. Nesta situação outro adulto do sexo masculino foi selecionado para participar da entrevista. Em 2005 a amostra final foi de 1089 domicílios, distribuídos nos 75 setores censitários e em 2010 a amostra final foi de 1121 domicílios, distribuídos nos mesmos setores censitários.

Para o presente estudo considerou-se dados de adolescentes de 12 a 18,9 anos. Nas duas pesquisas, foram considerados elegíveis os adolescentes que não apresentavam deficiência física que impedisse a avaliação antropométrica e aplicação dos questionários e que não estivessem grávidas. Em 2005, inicialmente, 561 adolescentes elegíveis foram entrevistados. Cinquenta adolescentes foram excluídos por inconsistência no preenchimento dos questionários, analisando-se os dados de 511 adolescentes (91,0\% dos elegíveis). Em 2010, os 314 adolescentes considerados elegíveis foram entrevistados (100\%).

Considerando prevalência estimada de excesso de peso em adolescentes de $20,5 \%{ }^{1}$, nível de confiança de $95 \%$, com precisão absoluta de 5\% para 2005 e de $4 \%$ para 2010 e o efeito do desenho por conglomerado, os números amostrais de 511 e 314, respectivamente, permitem estimar a prevalência de excesso de peso na população avaliada ${ }^{19}$.

A coleta de dados, tanto em 2005 como em 2010, foi realizada por equipe de entrevistadores devidamente treinados para as etapas de aplicação do questionário, avaliação de consumo alimentar e medidas antropométricas. A equipe era composta por nutricionistas ou estudantes de nutrição, cujo treinamento foi realizado em uma semana (40 horas totais), pela mesma equipe de coordenação dos dois inquéritos populacionais, de modo a manter a padronização e qualidade na coleta de dados. Especificamente para aferição de medidas antropométricas, a padronização da equipe foi feita com base nos critérios de Habi$\mathrm{cht}^{20}$. As entrevistas domiciliares e aferições de medidas foram realizadas mediante assinatura do termo de consentimento livre e esclarecido pelo responsável do domicílio e só participaram os adolescentes que quiseram. Os projetos foram aprovados pelos comitês de Ética e Pesquisa do Instituto de Medicina Social da Universidade Estadual do Rio de Janeiro e do Instituto de Estudos de Saúde Coletiva da Universidade Federal do Rio de Janeiro.

Em ambas as pesquisas foram aferidos peso e estatura com os indivíduos trajando roupas leves e descalços. Os equipamentos utilizados (balanças e estadiômetro portáteis) foram aferidos ao longo do período de coleta de dados para o controle de possíveis vieses de aferição. O peso foi obtido por meio de balança eletrônica portátil (Kratos PPS ${ }^{\circledR}$, São Paulo, Brasil), com capacidade até 150 quilogramas e variação de 50 gramas. A estatura foi medida por meio de estadiômetro portátil (Leicester ${ }^{\circledR}$, Reino Unido), com variação de 0,1 centímetros $(\mathrm{cm})$, sendo realizadas duas medidas, obtendo-se a média. Foi permitida uma variação máxima de $0,5 \mathrm{~cm}$ entre as duas medidas e caso a variação ultrapasse este valor as medidas eram repetidas. $\mathrm{O}$ peso e a estatura foram obtidos seguindo as técnicas propostas por Gordon et al. ${ }^{21}$. Com as medidas de peso e estatura calculou-se o Índice de Massa Corporal $\left(\mathrm{IMC}=\right.$ peso/estatura $\left.{ }^{2}\right)$. A classificação quanto à adequação de peso foi feita segundo pontos de corte específicos do IMC, por sexo e idade, conforme proposto pela Organização Mundial de Saúde ${ }^{22}$ : baixo IMC para idade $(<$ escore $-Z=-2)$; IMC adequado ( $\geq$ escore- $Z=-2$ e $<$ escore $-Z=+1$ ); sobrepeso ( $\geq$ escore $-Z=+1$ e $<$ escore $-Z=+2$ ); e obesidade ( $\geq$ escore- $Z=+2$ ). As categorias sobrepeso e obesidade foram agrupadas na categoria excesso de peso nas análises de associação.

A prevalência de insegurança alimentar foi investigada por meio da Escala Brasileira de Insegurança Alimentar (EBIA), adaptada e validada para a população brasileira ${ }^{23}$ e recomendada para uso em estudos populacionais ${ }^{24}$. A EBIA foi respondida pelo responsável pela alimentação da família e cada resposta afirmativa correspondeu a "um ponto", sendo o somatório do número de respostas afirmativas utilizado para a classificação quanto ao grau de insegurança alimentar (Segurança alimentar e insegurança alimentar leve, moderada e grave). Cabe ressaltar que a 
EBIA avalia Segurança Alimentar e Nutricional apenas pela dimensão do acesso à aquisição de alimentos; logo, o termo Segurança Alimentar se apresenta mais adequado, pois exclui a dimensão nutricional não avaliada pelo referido método ${ }^{25}$.

As variáveis demográficas avaliadas foram sexo, idade e cor da pele, esta última obtida por meio de autoavaliação com base nas opções utilizadas pelo IBGE ${ }^{26}$. Como indicador socioeconômico foi considerada a renda familiar mensal per capita (total de rendimentos da família dividido pelo número de moradores), expressa em múltiplos do salário mínimo: $\mathrm{R} \$ 300$ em 2005 e R\$ 510 em 2010.

A análise dos dados foi realizada utilizandose o software Statistical Program for the Social Sciences (SPSS versão 19,0, Chicago, IL, USA). Para as análises considerou-se o peso amostral de cada indivíduo para expansão da amostra e o efeito do desenho do estudo por conglomerados, utilizando-se o procedimento Complex Sample do SPSS.

Foi feita análise descritiva considerando as frequências e intervalos de confiança de 95\% (IC95\%) nos dois períodos (2005 e 2010) para as variáveis de desfecho (presença ou ausência de excesso de peso), e variáveis de exposição: sexo (masculino e feminino), idade (12 a 14,9 e 15 a 18,9 anos), cor da pele (preto ou pardo e branco), renda per capita (até $1 / 2$ salário mínimo, maior que $1 / 2$ salário e menor que 1 salário, e maior que 1 salário mínimo) e classificação da insegurança alimentar (variável em 4 categorias: segurança alimentar, insegurança alimentar leve, moderada e grave). As frequências foram comparadas entre as duas pesquisas com base no teste qui-quadrado. No caso das variáveis com mais de duas categorias foi realizado o teste de partição do qui-quadrado no programa estatístico WinPepi ${ }^{27}$. Investigou-se a associação entre as variáveis de desfecho e exposição, em cada período de estudo, com base no teste qui-quadrado. Utilizou-se regressão logística para amostras complexas a fim de verificar a associação entre as mudanças temporais de excesso de peso, segundo as variáveis sociodemográficas e a insegurança alimentar. Foi considerado nas análises o valor de $\mathrm{p}<0,05$ para significância estatística.

\section{Resultados}

Aproximadamente $80 \%$ dos adolescentes eram de cor preta ou parda e cerca de $50 \%$ tinham renda per capita de até 1 salário mínimo. Ade- mais, verificou-se redução do baixo peso $(4,5 \%$ para $0,9 \%)$ e aumento de sobrepeso (16,3\% para $22,8 \%)(p=0,033)$. Quanto à insegurança alimentar, não se verificou mudança significativa, todavia enquanto em 2005, aproximadamente, $60 \%$ dos adolescentes moravam em lares com insegurança alimentar, em 2010 essa prevalência passou para cerca de $50 \%$ (Tabela 1 ).

Nas análises estratificadas por sexo, verificouse que em 2010, entre os meninos, a prevalência de excesso de peso, foi maior entre os que tinham de 12 a 14,9 anos do que entre os de 15 a 18,9 anos ( $49,5 \%$ vs. $18,6 \%$, p=0,003). Em 2005 os meninos com renda per capita maior que 1 salário mínimo, apresentaram maior prevalência $(46,0 \%)$ de excesso de peso quando comparados aqueles com rendas entre $1 / 2$ e 1 salário $(25,2 \%)$ e menor que $1 / 2$ salário mínimo $(13,6 \%)(\mathrm{p}=0,012)$ (Tabela 2).

Quanto à evolução do excesso de peso pode se notar que nos meninos houve aumento significativo para os mais novos (20,1\% para $49,5 \%)$, os de cor preta ou parda (22,2\% para $37,3 \%)$, os que tinham renda per capita de até $1 / 2$ salário mínimo (13,6\%para $32,5 \%)$ e os que apresentavam insegurança alimentar moderada e grave $(9,2 \%$ para $36,3 \%$ ) entre 2005 e 2010 (Tabela 2).

Nas meninas, a prevalência de excesso de peso assim como a variação entre os dois períodos, não apresentou associação com as variáveis sociodemográficas e insegurança alimentar (Tabela 3).

\section{Discussão}

Ressalta-se como principais resultados do presente estudo o aumento de 7,5 pontos percentuais no excesso de peso entre 2005 e 2010, destacando-se que meninos mais novos, de cor preta ou parda, que tinham menor renda per capita e que apresentavam insegurança alimentar moderada e grave apresentaram maior aumento nesta prevalência entre os dois períodos investigados.

Ademais, foi verificada redução de 10,3 pontos percentuais na insegurança alimentar. Considerando a associação direta entre excesso de peso e insegurança alimentar já evidenciada em estudos com adolescentes ${ }^{7-9,12}$, poder-se-ia supor que a redução na insegurança alimentar observada neste estudo, estaria associada a redução do excesso de peso nos adolescentes. Entretanto, este resultado fortalece a questão de que, em países em desenvolvimento, a melhoria nas condições socioeconômicas, que podem estar relacionadas a 
Tabela 1. Características sociodemográficas, classificação de peso com base no IMC e insegurança alimentar dos adolescentes. Campos Elíseos, Duque de Caxias-RJ, 2005 e 2010.

\begin{tabular}{|c|c|c|c|c|c|c|}
\hline & \multicolumn{2}{|r|}{2005} & \multicolumn{2}{|r|}{2010} & \multirow{2}{*}{ Variação } & \multirow{2}{*}{$\begin{array}{c}\text { Valor de } \\
\mathbf{p}^{1}\end{array}$} \\
\hline & $n^{*}$ & $\%($ IC95\%) & $\mathbf{n}^{*}$ & $\%($ IC95\%) & & \\
\hline Sexo & 511 & & 314 & & & 0,668 \\
\hline Masculino & & $49,3(44,5-54,1)$ & & $51,0(44,3-57,7)$ & $+1,7$ & \\
\hline Feminino & & $50,7(45,9-55,5)$ & & $49,0(42,3-55,7)$ & $-1,7$ & \\
\hline Idade (anos) & 511 & & 314 & & & 0,178 \\
\hline 12 a 14,9 & & $44,9(39,3-50,6)$ & & $50,3(42,7-57,9)$ & $+5,4$ & \\
\hline 15 a 18,9 & & $55,1(49,4-60,7)$ & & $49,7(42,1-57,3)$ & $-5,4$ & \\
\hline Cor da pele & 510 & & 308 & & & 0,572 \\
\hline Preto/Pardo & & $81,2(76,7-85,0)$ & & $78,9(70,9-85,1)$ & $-2,3$ & \\
\hline Branco & & $18,8(15,0-23,3)$ & & $21,1(14,9-29,1)$ & $+2,3$ & \\
\hline Renda per capita ${ }^{\star *}$ & 503 & & 304 & & & 0,470 \\
\hline Até 1⁄2 salário mínimo & & $47,7(41,2-54,3)$ & & $50,5(40,7-60,2)$ & $+2,8$ & \\
\hline 1⁄2 - 1 salário mínimo & & $42,3(36,8-47,9)$ & & $36,5(28,5-45,3)$ & $-5,8$ & \\
\hline$>1$ salário mínimo & & $10,1(7,1-14,1)$ & & $13,1(7,4-22,1)$ & $+3,0$ & \\
\hline Classificação do IMC & 511 & & 301 & & & 0,033 \\
\hline Baixo peso & & $4,5(2,7-7,2)$ & & $0,9^{*}(0,3-2,8)$ & $-3,6$ & \\
\hline Peso adequado & & $72,2(66,9-76,9)$ & & $68,2(60,5-74,9)$ & $-4,0$ & \\
\hline Sobrepeso & & $16,3(12,7-20,6)$ & & $22,8^{\#}(17,3-29,5)$ & $+6,5$ & \\
\hline Obesidade & & $7,1(4,8-10,5)$ & & $8,1(5,1-12,8)$ & $+1,0$ & \\
\hline $\mathrm{IA}^{* * *}$ & 506 & & 314 & & & 0,051 \\
\hline Segurança alimentar & & $40,2(33,9-46,8)$ & & $50,5(41,1-59,8)$ & $+10,3$ & \\
\hline IA leve & & $35,6(30,1-41,5)$ & & $35,0(27,5-43,3)$ & $-0,6$ & \\
\hline IA moderada e grave & & $24,2(19,4-29,8)$ & & $14,5(9,2-22,0)$ & $-9,7$ & \\
\hline
\end{tabular}

Fonte: Elaborado pelos autores.

melhoria na insegurança alimentar, sem um processo de maior acesso à educação, em populações tradicionalmente mais pobres, pode repercutir na aquisição de alimentos de maior densidade energética $^{28,29}$, porém de menor custo e, consequentemente, em aumento do excesso de peso.

Neste contexto, Segall-Corrêa e Salles-Cos$\mathrm{ta}^{28}$, em estudo de base populacional realizado com 5 mil famílias de todo o território nacional, encontraram percentual importante de famílias que, após serem incluídas no programa de transferência de renda de nosso país, o Programa Bolsa família (PBF), referiram aumento significativo na quantidade e variedade de alimentos consumidos. Entretanto, as autoras frisaram que o aumento do poder de compra não refletiu em benefício na qualidade da dieta porque houve aumento no consumo de alimentos de alta densidade energética. Lignani et al. ${ }^{29}$, notaram ainda que as famílias residentes em Duque de Caxias, relataram aumento do consumo de todos os grupos alimentares, principalmente cereais, alimentos processados, carne, leite e produtos lácteos, e açúcar a partir da entrada no PBF. Além disso, o grau de dependência de rendimentos provenientes do programa foi, positivamente, associado com aumento da ingestão de açúcar e refrigerantes.

Os estudos acima ressaltam a importância de que medidas de educação nutricional sejam realizadas em conjunto com a melhoria no acesso aos alimentos. Entretanto, Henriques et al. ${ }^{30}$ destacam a influência do ambiente obesogênico, ao qual a população está exposta. Este ambiente envolve a oferta, disponibilidade, conveniência e praticidade de preparo dos alimentos, relação custo saciedade, a hiperpalatabilidade dos produtos ultraprocessados, diferentes estratégias de marketing envolvendo estes alimentos, além de outros aspectos. Neste contexto, esses fatores podem interferir no processo decisório em torno da alimentação. Nota-se assim que a relação entre insegurança alimentar e obesidade é complexa e, 
Tabela 2. Prevalência de excesso de peso, segundo as variáveis sociodemográficas e insegurança alimentar, nos adolescentes do sexo masculino. Campos Elíseos, Duque de Caxias-RJ, 2005 e 2010.

\begin{tabular}{|c|c|c|c|c|c|c|c|}
\hline & \multicolumn{6}{|c|}{ Excesso de peso } & \multirow{3}{*}{$\begin{array}{c}\text { Variação } \\
\%\end{array}$} \\
\hline & \multicolumn{3}{|c|}{2005} & \multicolumn{3}{|c|}{2010} & \\
\hline & $\mathbf{n}^{*}$ & $\%(\mathrm{IC} 95 \%)$ & p & $\mathbf{n}^{*}$ & $\%($ IC95\%) & $\mathbf{p}$ & \\
\hline Idade (anos) & & & 0,589 & & & 0,003 & \\
\hline 12 a 14,9 & 112 & $20,1(12,1-31,5)$ & & 80 & $49,5(35,1-64,0)$ & & $+29,4^{\#}$ \\
\hline 15 a 18,9 & 140 & $23,9(16,4-33,4)$ & & 74 & $18,6(10,1-31,9)$ & & $-5,3$ \\
\hline Cor/Raça & & & 0,951 & & & 0,352 & \\
\hline Preto/Pardo & 204 & $22,2(15,9-30,0)$ & & 119 & $37,3(25,8-50,4)$ & & $+15,1^{\#}$ \\
\hline Branco & 47 & $22,7(11,9-38,9)$ & & 34 & $26,0(12,3-46,9)$ & & $+3,3$ \\
\hline Renda per capita ${ }^{* *}$ & & & 0,012 & & & 0,638 & \\
\hline Até $1 / 2$ salário mínimo & 105 & $13,6(8,0-22,2)$ & & 65 & $32,5(19,5-49,0)$ & & $+18,9^{\#}$ \\
\hline $1 / 2-1 S M$ & 114 & $25,2(16,3-36,7)$ & & 63 & $41,2(25,6-58,8)$ & & $+16,0$ \\
\hline$>1$ salário mínimo & 25 & $46,0(23,9-69,9)$ & & 18 & $26,8(7,8-61,2)$ & & $-19,2$ \\
\hline Insegurança alimentar & & & 0,109 & & & 0,986 & \\
\hline Segurança alimentar & 105 & $25,8(16,5-37,8)$ & & 73 & $34,0(21,6-49,0)$ & & $+8,2$ \\
\hline Insegurança alimentar leve & 94 & $24,8(15,7-36,8)$ & & 50 & $34,8(18,9-55,1)$ & & $+10,0$ \\
\hline $\begin{array}{l}\text { Insegurança alimentar moderada } \\
\text { e grave }\end{array}$ & 50 & $9,2(3,7-21,2)$ & & 29 & $36,3(16,5-62,1)$ & & $+27,1^{\#}$ \\
\hline
\end{tabular}

*Os números diferem devido a perdas em cada variável. ${ }^{* *}$ Valor do salário mínimo: $2005=\mathrm{R} \$ 300,00 ; 2010=\mathrm{R} \$ 510,00$.

"Regressão logística, $\mathrm{p}<0,05$.

Fonte: Elaborado pelos autores.

embora ações de educação alimentar e nutricional tenham papel importante para a promoção de alimentação adequada e saudável, outras medidas estruturais são necessárias, como apontam Cotta e Machado ${ }^{31}$.

Em relação a prevalência de excesso de peso encontrada no presente estudo, os adolescentes apresentaram em 2010, maior prevalência $(30,9 \%)$ que a encontrada pela Pesquisa de Orçamentos Familiares (POF 2008-2009) na região Sudeste $(22,6 \%)^{1}$, e menor em relação à Pesquisa Nacional de Saúde do Escolar (PeNSE 2015) ${ }^{32}$, que foi de $33,1 \%$ para mesma região. Tais achados podem indicar que, mesmo em área caracterizada por evidente situação de insegurança alimentar, os jovens apresentam prevalência de excesso de peso superior ou similar à média encontrada para os adolescentes brasileiros. Em contrapartida, no estudo de Guerra et al..$^{33}$, realizado com adolescentes da área da Amazônia Legal (Mato Grosso), também marcada pela prevalência elevada de insegurança alimentar, foi verificada prevalência de excesso de peso menor $(13,8 \%)$ quando comparada com este estudo, e inclusive, inferior à da média nacional para a região Centro-Oeste $(21,9 \%)^{1}$, no mesmo período das pesquisas de Duque de Caxias (Rio de Janeiro). Com isso, novos estudos precisam ser rea- lizados a fim de verificar as diferenças regionais nas prevalências de excesso de peso em áreas de insegurança alimentar no Brasil.

Vale destacar também que o sexo foi uma variável diferencial nas associações investigadas. Enquanto entre os meninos faixa etária, cor da pele, renda e insegurança alimentar foram associadas ao excesso de peso e sua evolução, nas meninas nenhuma associação foi observada. $\mathrm{O}$ fato dos adolescentes do sexo masculino mais novos (12 a 14 anos) apresentarem maior prevalência e aumento significativo do excesso de peso, corrobora com os achados da POF 2008-2009' ${ }^{1}$, na qual observou-se que o excesso de peso foi justamente mais elevado na fase inicial da adolescência. Esse panorama gera grande preocupação, já que cada vez mais cedo os jovens têm apresentado excesso de peso, fator que tem sido associado ao surgimento precoce de alterações metabólicas tais como a pressão arterial elevada, diabetes mellitus, doenças cardiovasculares e síndrome metabólica $^{34}$. Cabe destacar que tais alterações já foram observadas em adolescentes brasileiros com excesso de peso ${ }^{35,36}$.

Em relação à renda, na POF 2008-2009, estudo realizado na mesma época que o nosso, verificouse que adolescentes de camadas econômicas menos favorecidas apresentam menores prevalências 
Tabela 3. Prevalência de excesso de peso, segundo as variáveis sociodemográficas e insegurança alimentar, nos adolescentes do sexo feminino. Campos Elíseos, Duque de Caxias-RJ, 2005 e 2010.

\begin{tabular}{|c|c|c|c|c|c|c|c|}
\hline & \multicolumn{6}{|c|}{ Excesso de peso } & \multirow{3}{*}{$\begin{array}{c}\text { Variação } \\
\%\end{array}$} \\
\hline & \multicolumn{3}{|c|}{2005} & \multicolumn{3}{|c|}{2010} & \\
\hline & $\mathbf{n}^{\star}$ & $\%(\mathrm{IC} 95 \%)$ & $\mathbf{p}$ & $\mathbf{n}^{*}$ & $\%(\mathrm{IC} 95 \%)$ & $\mathbf{p}$ & \\
\hline Idade (anos) & & & 0,400 & & & 0,636 & \\
\hline 12 a 14,9 & 118 & $27,8(18,0-40,3)$ & & 68 & $29,4(18,1-44,1)$ & & $+1,6$ \\
\hline 15 a 18,9 & 141 & $21,8(15,0-30,6)$ & & 80 & $25,1(14,8-39,3)$ & & $+3,3$ \\
\hline Cor/Raça & & & 0,070 & & & 0,932 & \\
\hline Preto/Pardo & 210 & $27,0(20,3-35,0)$ & & 120 & $27,2(18,3-38,4)$ & & $+0,2$ \\
\hline Branco & 49 & $13,8(6,4-27,1)$ & & 28 & $28,0(14,3-47,4)$ & & $+14,2$ \\
\hline Renda per capita ${ }^{\star *}$ & & & 0,077 & & & 0,386 & \\
\hline Até $1 / 2$ salário mínimo & 135 & $30,9(22,7-40,5)$ & & 78 & $29,1(17,5-44,2)$ & & $-1,8$ \\
\hline $1 / 2-1 S M$ & 98 & $18,6(11,2-29,3)$ & & 46 & $16,0(5,6-37,9)$ & & $-2,6$ \\
\hline$>1$ salário mínimo & 26 & $14,1(4,7-35,5)$ & & 22 & $37,1(13,3-69,2)$ & & $+23,0$ \\
\hline Insegurança alimentar & & & 0,411 & & & 0,824 & \\
\hline Segurança alimentar & 99 & $24,3(14,6-37,6)$ & & 79 & $25,0(14,7-39,4)$ & & $+0,7$ \\
\hline Insegurança alimentar leve & 86 & $19,6(12,5-29,3)$ & & 54 & $28,0(14,5-47,2)$ & & $+8,4$ \\
\hline $\begin{array}{l}\text { Insegurança alimentar moderada } \\
\text { e grave }\end{array}$ & 73 & $31,2(19,3-46,3)$ & & 16 & $33,8(14,0-61,6)$ & & $+2,6$ \\
\hline
\end{tabular}

${ }^{*}$ Os números diferem devido a perdas em cada variável. ${ }^{* *}$ Valor do salário mínimo: $2005=\mathrm{R} \$ 300,00 ; 2010=\mathrm{R} \$ 510,00$.

Fonte: Elaborado pelos autores.

de excesso de peso em comparação com adolescentes de nível socioeconômico mais elevado, particularmente entre os meninos ${ }^{1}$. Em pesquisa com estudantes do ensino médio de escolas da região metropolitana do Rio de janeiro, Moreira et al..$^{37}$, verificaram maior ganho de IMC entre meninos de escolas particulares quando comparados aos de escolas públicas. No presente estudo o excesso de peso aumentou de modo significativo, justamente entre os meninos mais pobres, o que vai no sentido oposto aos achados citados anteriormente. Entretanto, alguns autores têm verificado que a relação excesso de peso e nível socioeconômico pode estar mudando em países de baixo e médio nível socioeconômico, com importantes prevalências de excesso de peso em indivíduos de nível socioeconômico menos favorecido ${ }^{38,39}$.

A evolução de excesso de peso também foi maior entre os meninos de cor preta ou parda, e sabe-se que, em nosso país esta é uma variável mais relacionada ao contexto social do que ao fator biológico propriamente dito ${ }^{37,40}$. Além disso, é possível notar que o excesso de peso aumentou de forma significativa justamente entre os meninos com níveis de insegurança alimentar moderado e grave. Salles-Costa et al. ${ }^{16}$, estudando a população residente em Caxias em 2005, verificaram que dentre as famílias com renda mensal per capita menor que $1 / 4$ do salário-mínimo, $88,0 \%$ relataram algum grau de insegurança alimentar. Notaram também que na medida em que a renda familiar diminuiu, a proporção de famílias em insegurança alimentar aumentou. Essa associação verificada entre renda e insegurança alimentar indica que, para esses adolescentes, o aumento do excesso de peso está fortemente associado com os níveis socioeconômicos menos favorecidos, que nos instiga a tentar entender o motivo dessa associação.

Sabe-se que a situação de insegurança alimentar vivenciada na adolescência é relevante uma vez que essa fase é marcada por intenso desenvolvimento físico, cognitivo e emocional ${ }^{41}$. É importante o acesso contínuo à alimentação, em quantidade suficiente e qualidade adequada, para garantir o bom crescimento e desenvolvimento ${ }^{42}$. Neste estudo constatou-se que a prevalência de segurança alimentar aumentou enquanto os níveis mais graves de insegurança reduziram entre 2005 e 2010. Uma justificativa pode ser o maior acesso a programas de transferência de renda como o PBF. Este fato pode ter auxiliado de forma definitiva na redução das prevalências dos graus mais graves de insegurança alimentar. Palmeira et al. ${ }^{43}$ concluíram que este tipo de programa pode contribuir para reduzir a insegurança 
alimentar em populações vulneráveis à pobreza. Entretanto, vale ressaltar o cenário atual de retrocesso dos programas sociais e recrudescimento de situações mais graves de insegurança alimen$\operatorname{tar}^{44}$, o que pode apresentar impacto negativo no local onde o estudo foi realizado.

Os resultados deste estudo devem ser analisados à luz de suas limitações e pontos fortes. Quanto as primeiras, a reflexão inicial consiste no fato do conceito de insegurança alimentar, aferido pela EBIA, não corresponder ao conceito integral ampliado de segurança alimentar e nutricional (SAN) adotado pelo Brasil ${ }^{45}$, entretanto consiste em instrumento de aferição deste desfecho validado no país desde 2003 com base na escala norte-americana de insegurança alimentar ${ }^{23}$. Ademais, a EBIA tem demonstrado ser psicometricamente válida ${ }^{46}$, reforçando sua adequação no monitoramento de insegurança alimentar por meio de estudos de prevalência, identificando populações em risco e no estudo de causas como a pobreza ${ }^{47}$. Nesse sentido, é possível pensar na relação do consumo alimentar destes adolescentes, que reflete a dimensão ampliada do conceito de SAN, a fim de verificar a associação entre o aumento do excesso de peso e a insegurança alimentar observado no presente estudo.

Cabe destacar ainda que, por ser uma pesquisa realizada a partir de dois estudos transversais, apresenta a limitação de não permitir determinar a causalidade das associações entre o excesso de peso e as variáveis estudadas. Além disso, o poder

\section{Colaboradores}

DD Santana participou da concepção, análises estatísticas e redação do artigo. EG Barros participou das análises estatísticas. R Salles-Costa participou da concepção do estudo e da redação final do artigo. GV Veiga participou da concepção do estudo, das análises estatísticas e redação final do artigo. da amostra não foi calculado para verificar a variação de excesso de peso entre as duas pesquisas e para a abordagem com as análises estratificadas, mas teve-se a preocupação de calcular o poder da amostra em cada período para estimativa de prevalência de excesso de peso nos adolescentes.

Como ponto forte destaca-se a avaliação de amostra representativa de adolescentes residentes em área de alta vulnerabilidade social, diferente da maioria dos estudos que avaliam populações mais heterogêneas em relação à classe social. Assim, o estudo pode auxiliar e esclarecer de forma mais específica a evolução de excesso de peso entre estes adolescentes.

Conclui-se assim que, o aumento da prevalência de excesso de peso foi expressivo nos adolescentes residentes em área de baixo nível socioeconômico e alta prevalência de insegurança alimentar, em período de 5 anos, sendo os meninos mais novos, pretos ou pardos, de menor renda e residentes em lares com insegurança alimentar moderada e grave mais expostos a este aumento. Assim, no contexto atual em que se discute a sinergia entre as pandemias de obesidade, desnutrição e as mudanças climáticas, questões que apresentam causas comuns ${ }^{48}$, os resultados deste estudo contribuem para reforçar a necessidade de políticas públicas necessárias para melhoria do panorama encontrado, focalizadas nesse estrato etário da população, visando o controle e o combate à obesidade em grupos expostos a pobreza e a insegurança alimentar.

\section{Financiamento}

Conselho Nacional de Desenvolvimento Científico e Tecnológico (CNPq) e Instituto Nacional de Câncer. 


\section{Referências}

1. Instituto Brasileiro de Geografia e Estatística (IBGE) Pesquisa de Orçamentos Familiares 2008-2009: Antropometria e estado nutricional de crianças, adolescentes e adultos no Brasil. Rio de Janeiro: IBGE; 2010.

2. Instituto Brasileiro de Geografia e Estatística (IBGE). Pesquisa nacional de saúde: 2013: ciclos de vida: Brasile grandes regiões. Rio de Janeiro: IBGE; 2015.

3. World Health Organization (WHO). Global Nutrition Report, 2017 [Internet]. [acessado 2019 abr 3]. Disponível em: https://globalnutritionreport.org/reports/2017-global-nutrition-report/.

4. Brasil. Lei ${ }^{\circ}$. 11.346, de 15 de setembro de 2006. Cria o Sistema Nacional de Segurança Alimentar e Nutricional - SISAN com vistas em assegurar o direito humano à alimentação adequada e dá outras providências. Diário Oficial da União; 2006.

5. Conselho Nacional de Segurança Alimentar (CNSA). Documento base da III Conferência Nacional de Segurança Alimentar e Nutricional. Brasília: CNSA; 2007.

6. Casey PH, Simpson PM, Gossett JM, Bogle ML, Champagne CM, Connell C, Harsha D, McCabe-Sellers B, Robbins JM, Stuff JE, Weber J. The association of child and household food insecurity with childhood overweight status. Pediatrics 2006; 118(5):1406-1413.

7. Gulliford MC, Nunes C, Rocke B. Food insecurity, weight control practices and body mass index in adolescents. Public Health Nutr 2006; 9(5):570-574.

8. Olson CM, Bove CF, Miller EO. Growing up poor: long-term implications for eating patterns and body weight. Appetite 2007; 49(1):198-207.

9. Holben DH, Taylor CA. Food Insecurity and Its Association With Central Obesity and Other Markers of Metabolic Syndrome Among Persons Aged 12 to 18 Years in the United States. J Am Osteopath Assoc 2015; 115(9):536-543.

10. Drewnowski A. Obesity, diets, and social inequalities. Nutr Rev 2009; 67(Supl. 1):S36-S39.

11. Knol LL, Haughton B, Fitzhugh EC. Food insufficiency is not related to the overall variety of foods consumed by young children in low-income families. $J \mathrm{Am}$ Diet Assoc 2004; 104(4):640-644.

12. Santos JV, Gigante DP, Domingues MR. Prevalência de insegurança alimentar em Pelotas, Rio Grande do Sul, Brasil, e estado nutricional de indivíduos que vivem nessa condição. Cad Saude Publica 2010; 26(1):41-49.

13. Vicenzi K, Henn RL, Weber AP, Backes V, Paniz VMV, Donatti T, Olinto MTA. Insegurança alimentar e excesso de peso em escolares do primeiro ano do Ensino Fundamental da rede municipal de São Leopoldo, Rio Grande do Sul, Brasil. Cad Saude Publica 2015; 31(5):1084-1094.

14. Lopes T, Veiga GV, Salles-Costa R, Costa RS, Sichieri R, Pereira RA. Family food insecurity and nutritional risk in adolescents from a low-income area evaluated in a population-based cross-sectional study. J Biosoc Sci 2013; 45(5):661-674

15. Oliveira JS, Lira PIC, Veras ICL, Maia SR, Lemos MCC, Andrade SLLS, Viana Junior MJ, Pinto FCL, Leal VS, Batista Filho M. Estado nutricional e insegurança alimentar de adolescentes e adultos em duas localidades de baixo índice de desenvolvimento humano. Rev Nutr 2009; 22(4):453-465.
16. Salles-Costa R, Pereira RA, Vasconcellos MTL, Veiga GV, Marins VMR, Jardim BC, Gomes FS, Sichieri R. Associação entre fatores socioeconômicos e insegurança alimentar: estudo de base populacional na região metropolitana do Rio de Janeiro, Brasil. Rev Nutr 2008; 21(Supl.):99-109.

17. Ferreira JES, Souza PRB, Costa RS, Sichieri R, Veiga GV. Disordered eating behaviors in adolescents and adults living in the same household in metropolitan area of Rio de Janeiro, Brazil. Psychiatry Res 2013; 210(2):612-617.

18. Haldane JBS. On a method of estimating frequencies. Biometrika 1945; 3:222-225.

19. Lwanga SK, Lemeshow S. Sample Size Determination in Health Studies: A Practical Manual. Geneva: WHO; 1991.

20. Habicht JP. Estandarizacion de metodos epidemiológicos cuantitativos sobre el terreno. Bol Oficina Sanit Panam 1974; 76(5):375-384.

21. Gordon CC, Chumlea WC, Roche AF. Stature, Recumbent Length and Weight. In: Lohman TG, Roche AF, Martorell R. Anthropometric Standardization Reference Manual. Champaign: Human Kinetics Books; 1988. p. 3-8.

22. Onis M, Onyango AW, Borghi E, Siyam A, Nishida C, Siekmann J. Development of a WHO growth reference for school-aged children and adolescents. Bull World Health Organ 2007; 85(9):660-667.

23. Pérez-Escamilla R, Segall-Corrêa AM, Kurdian ML, Sampaio MD, Marín-León L, Panigassi G. An Adapted Version of the U.S. Department of Agriculture Food Insecurity Module Is a Valid Tool for Assessing Household Food Insecurity in Campinas, Brazil. J Nutr 2004; 134(8):1923-1928

24. Salles-Costa R. Diagnóstico de insegurança alimentar nos estudos populacionais. In: Schneider OF. Segurança alimentar e nutricional: tecendo a rede de saberes. Rio de Janeiro: Faperj; 2012. p. 121-138.

25. Antunes MM, Sichieri R, Salles-Costa R. Consumo alimentar de crianças menores de três anos residentes em área de alta prevalência de insegurança alimentar domiciliar. Cad Saude Publica 2010; 26(8):1642-1650.

26. Instituto Brasileiro de Geografia e Estatística (IBGE). Estudo Nacional de Despesas Familiares: 1974-1975. Rio de Janeiro: IBGE; 1977.

27. Abramson JH. WINPEPI (PEPI-for-Windows): computer programs for epidemiologists. Epidemiol Perspect Innov 2004; 1(1):6.

28. Segall-Corrêa AM, Salles-Costa R. Novas possibilidades de alimentação a caminho? Democracia Viva 2008; 39:68-73.

29. Lignani JB, Burlandy L, Sichieri R, Salles-Costa R, Costa RS. Changes in food consumption among the Programa Bolsa Família participant families in Brazil. Public Health Nutr 2011; 14(5):785-792.

30. Henriques P, O'Dwyer G, Dias P, Barbosa R, Burlandy L. Políticas de Saúde e de Segurança Alimentar e Nutricional: desafios para o controle da obesidade infantil. Cien Saude Colet 2018; 23(12):4143-4152.

31. Cotta RMM, Machado JC. Programa Bolsa Família e segurança alimentar e nutricional no Brasil: revisão crítica da literatura. Revista Panam Salud Publica 2013; 33(1):54-60. 
32. Instituto Brasileiro de Geografia e Estatística (IBGE). Pesquisa nacional de saúde do escolar: 2015. Rio de Janeiro: IBGE; 2016.

33. Guerra LDS, Espinosa MM, Bezerra ACD, Guimarães LV, Lima-Lopes MA. Insegurança alimentar em domicílios com adolescentes da Amazônia Legal Brasileira: prevalência e fatores associados. Cad Saude Publica 2013; 29(2):335-348.

34. Malik VS, Willett WC, Hu FB. Global obesity: trends, risk factors and policy implications. Nat Rev Endocrinol 2013; 9(1):13-27.

35. Seki M, Matsuo T, Carrilho AJ. Prevalence of metabolic syndrome and associated risk factors in Brazilian schoolchildren. Public Health Nutr 2009; 12(7):947952.

36. Rizzo AC, Goldberg TB, Silva CC, Kurokawa CS, Nunes HR, Corrente JE. Metabolic syndrome risk factors in overweight, obese, and extremely obese Brazilian adolescents. Nutr J 2013; 12:19.

37. Moreira NF, Sichieri R, Reichenheim ME, Oliveira AS, Veiga GV. The associations of BMI trajectory and excessive weight gain with demographic and socio-economic factors: the Adolescent Nutritional Assessment Longitudinal Study cohort. Br J Nutr 2015; 114(12):2032-2038.

38. Dinsa GD, Goryakin Y, Fumagalli E, Suhrcke M. Obesity and socioeconomic status in developing countries: a systematic review. Obes Rev 2012; 13(11):10671079.

39. Monteiro CA, Moura EC, Conde WL, Popkin BM. Socioeconomic status and obesity in adult populations of developing countries: a review. Bull World Health Organ 2004; 82(12):940-946.

40. Ferreira MF, Moraes CL, Reichenheim ME, Verly E, Marques ES, Salles-Costa R. Effect of physical intimate partner violence on body mass index in low-income adult women. Cad Saude Publica 2015; 31(1):161-172.

41. Pérez-Escamilla R, Vianna RPT. Food insecurity and the behavioral and intellectual development of children: A review of the evidence. J Applied Res Children 2012; 3:1.

42. Rogol AD, Clark PA, Roemmich JN. Growth and pubertal development in children and adolescents: Effects of diet and physical activity. Am J Clin Nutr 2000; 72(2 Supl.):521S-528S.

43. Palmeira PA, Bem-Lignani J, Maresi VA, Mattos RA, Interlenghi G, Salles-Costa R. Temporal Changes in the Association Between Food Insecurity and Socioeconomic Status in Two Population-Based Surveys in Rio de Janeiro, Brazil. Soc Indic Res 2019; 144:1349.

44. Sousa L, Segall-Corrêa A, Ville A, Melgar-Quiñonez H. Food security status in times of financial and political crisis in Brazil. Cad Saude Publica 2019; 35(7):e00084118.
45. Segall-Correa AM, Perez-Escamilla R, Marin-Leon L, Yuyama L, Vianna RPT, Coitinho D, Sampaio MFA, Panigassi G, Maranha LK, Gubert M, Leão MM, Guimarães LV, Bergamasco SMPP, Oliveira JA, Corrêa MLM. Evaluation of household food insecurity in Brazil: validity assessment in diverse sociocultural settings. In: Initiativa América Latina y Caribe sin Hambre. Santiago: Oficina Regional FAO-Chile; 2009. p. 80-101.

46. Reichenheim ME, Interlenghi GS, Moraes CL, SegallCorrêa AM, Pérez-Escamilla R, Salles-Costa R. A model-based approach to identify classes and respective cut-offs of the Brazilian household food insecurity measurement scale. J Nutr 2016; 146(7):1356-1364.

47. Palmeira PA, Salles-Costa R, Pérez-Escamilla R. Effects of family income and conditional cash transfers on household food insecurity: evidence from a longitudinal study in Northeast Brazil. Public Health Nutr 2020; 23(4):756-767.

48. Swinburn BA, Kraak VI, Allender S, Atkins V, Baker P, Bogard J, Brinsden H, Calvillo A, De Schutter O, Devarajan R, Ezzati M, Friel S, Goenka S, Hammond RA, Hastings G, Hawkes C, Herrero M, Hovmand PS, Howden M, Jaacks LM, Kapetanaki AB, Kasman M, Kuhnlein HV, Kumanyika SK, Larijani B, Lobstein T, Long MW, Matsudo VKR, Mills SDH, Morgan G, Morshed A, Nece PM, Pan A, Patterson DW, Sacks G, Shekar M, Simmons GL, Smit W, Tootee A, Vandevijvere S, Waterlander WE, Wolfenden L, Dietz WH. The Global Syndemic of Obesity, Undernutrition, and Climate Change: The Lancet Commission report. Lancet 2019; 393(10173):791-846.

Artigo apresentado em 24/03/2020

Aprovado em 19/10/2020

Versão final apresentada em 21/10/2020

Editores-chefes: Romeu Gomes, Antônio Augusto Moura da Silva 